\title{
Developments in GDN Research: Introduction
}

\author{
Christof Weinhardt • Stefan Seifert
}

Published online: 11 December 2009

(C) Springer Science+Business Media B.V. 2009

Negotiations are omnipresent both in private and professional life, and many decisions are not made by individuals but result from the interaction of a group of agents. Depending on the context, negotiations can be driven by conflicting goals, incomplete information, misunderstandings, or simply by the desire to indentify the group's joint preferences. Accordingly, Group Decision and Negotiation research is a rich and varied field. It applies concepts and methods of diverse disciplines, including-but not limited to-information systems, computer science, game theory, and statistics. Moreover, many of the contributions to the field are themselves interdisciplinary.

The yearly meetings of the INFORMS section on Group Decision and Negotiation and the EURO Working Group on Group Decision and Negotiation Support reflect the most recent trends and developments on a regular basis. The 2006 conference in Karlsruhe drew about 80 papers covering methodological advancements and empirical studies as well as software systems that support negotiating parties in identifying mutual agreement.

This special issue comprises six papers selected from the contributions presented at the GDN 2006 meeting. The selection was based on an extensive review process following the conference. In particular, the papers also highlight the methodological and conceptual diversity of the field of Group Decision and Negotiation.

The first paper by Ofir Turel is both methodological and didactic. Turel argues that the data from observing (pairs of) negotiators is not independent. He also claims that this interdependence is largely neglected in group decision and negotiation research.

\footnotetext{
C. Weinhardt $\cdot$ S. Seifert $(\varangle)$

Karlsruhe Institute of Technology, Institute of Information Systems and Management, Englerstr. 14, 76131 Karlsruhe, Germany

e-mail: stefan.seifert@kit.edu

C. Weinhardt

e-mail: christof.weinhardt@kit.edu
} 
Failure to appropriately take dependencies in the data into account, however, could lead to inaccurate $p$-values with respect to statistical hypothesis tests even if parameter estimates are not biased. Moreover, interesting relations within or between dyads could be overlooked. To illustrate this point, Turel presents several models of interdependence on a conceptual level and either discusses how these interdependencies can be treated or refers the reader to the respective literature. In particular, Turel promotes the application of sub-sampling techniques and the estimation of multi-level modes as well as the actor-partner interdependence model in negotiation research, for which he also gives an example using a data set from the Inspire system.

Guoming Lai and Katia Sycara take a conceptual approach and develop a negotiation support system framework that facilitates the simultaneous negotiation of multiple attributes. Reviewing the existing literature, the authors claim, e.g., that artificial intelligence research is often based on simplified assumptions with respect to the information on the negotiators' utility functions. Moreover, game theoretical approaches dealing with these aspects abstract from requirements of actual implementations. Therefore, the framework presented by Lai and Sycara seeks to integrate the two disciplines and takes incomplete information, Pareto efficiency, and tractability explicitly into account. Building on asymmetric, complete and transitive preferences, and a negotiation protocol of alternating offers, the authors present a set of negotiation strategies that are suitable for an automated negotiation agent. These strategies can be broken down into conceding, responding, and proposing components and can also be applied if a negotiator has not elicited its own utility function prior to the negotiation.

Eva Chen and Ilka Weber stress the importance of the design of systems which facilitate negotiations. They investigate bidders' perceptions in an auction by means of a laboratory experiment. In the experiment a second price auction is compared with a related mechanism that grants a discount to selected bidders. The results show that the variation of the mechanism has a significant impact on both the behavior of the bidders and the outcome of the auctions. Despite the differences in the outcomes and the individual payoffs, the discount, however, does not influence the users' subjective perceptions, beliefs, or intentions vis-à-vis using the system. The authors thus question the importance of traditional mechanism design and conclude that in actual applications the design of the system is more relevant for the success of an auction platform than the design of the negotiation mechanism or its protocol.

Majid Sheikhmohammady, D. Marc Kilgour, and Keith W. Hipel investigate an actual political negotiation problem. They apply various concepts from the social choice literature and fallback bargaining procedures as well as bankruptcy rules to the conflict between Azerbaijan, Iran, Kazakhstan, Russia, and Turkmenistan over the legal status of the Caspian Sea. As the seabed is rich in oil and natural gas reserves, the stakes in this conflict are high. At the same time, the solution is difficult because the negotiators have different or even opposing preferences regarding the possible options for resolution. Sheikhmohammady et al. show that theoretical predictions of a negotiation outcome depend on which theoretical concepts are applied: Different social choice procedures favor the division of the sea based on historical Soviet maps, but bankruptcy allocation rules propose a proportional allocation of resources. The proportional allocation is somewhat similar to the predictions of fallback bargaining, which suggest a division based on the International Law of the Seas. Interestingly, 
fallback bargaining also shows that no agreement at all is a likely outcome if "impasse" is considered as one of the options. Possibly, this is one of the reasons why the conflict has not yet been settled.

Bernado A. Huberman and Dennis M. Wilkinson extend the analysis of negotiations to the broader context of interdependencies in collaborative work. They extend the work transformation matrix (WTM) to a model that captures stochastic fluctuations in the progress of individual tasks. It is shown that the pure existence of perturbations can cause delays in a joint project. Moreover, these delays increase with the stochastic noise. The model can serve as a tool for managers to identify the main drivers for the risk of running behind schedule, so that appropriate measures for mitigation may be taken well ahead of time. Furthermore, it allows the comparison of alternative project organizations with respect to average completion time. A rather general result is that long delays tend to be less likely in hierarchical organizations as opposed to flat ones. The paper adds two important contributions to Group Decision and Negotiations research. First, the latter result can be applied to negotiations in a straightforward manner: It suggests that complex negotiations could be shortened if individual packages are delegated to particular task forces. Second, one of the main reasons for fluctuations in project progress is miscommunication between individual teams, which in turn implies that fluctuations (and thus expected project delays) could be reduced by improving communication.

Improving the quality of communication within a negotiation process is also the focus of the last paper in this issue, written by Mareike Schoop, Frank Köhne, and Katja Ostertag. The authors argue that the quality of a negotiation refers not only to its outcome, but also to the communication process upon which the result is based. Business relationships evolve from the very start of the negotiation, and therefore it is particularly important to investigate the whole communication process leading up to the agreement. In fact, the authors conjecture that communication quality negatively affects transaction costs and suggest further research to test this hypothesis. In their paper, Schoop et al. elaborate on common concepts of (communication) quality in different disciplines such as social psychology, marketing, or information systems research, and derive requirements for high-quality communication, particularly in business negotiations. With respect to these requirements, they introduce a definition of communication quality that integrates views on the semiotic level, the interactive process, and the relational context of a negotiation. In order to measure communication quality along the dimensions of effectiveness, efficiency, and relationship management, a mix of methods capturing both subjective and objective aspects as well as the interactive and dynamic nature of a negotiation is suggested.

Acknowledgements We thank the authors for their contributions to this issues as well as the reviewers for their support and valuable suggestions for improvement. We hope you will find reading the papers interesting and inspiring. 